No. 2

\title{
ONE-DIMENSIONAL FALICOV-KIMBALL MODEL IN THE STRONG COUPLING LIMIT
}

\author{
P. FARKašovskÝ aNd I. BaT'Ko \\ Institute of Experimental Physics, Slovak Academy of Sciences \\ Watsonova 47, 04353 Košice, Slovakia.
}

\begin{abstract}
We studied the ground state properties of the one-dimensional FalicovKimball model in the strong coupling limit for special periodic and aperiodic configurations of ions. The ground state phase diagrams of the model for nearest neighbor and next nearest neighbor hopping are discussed.
\end{abstract}

PACS numbers: 75.10.Lp

The Falicov-Kimball model [1] can be considered as the quantum mechanical lattice model of electrons interacting with classical ions. It is defined by the second-quantized Hamiltonian

$$
H=\sum_{i, j} t_{i j} c_{i}^{+} c_{j}+U \sum_{i} w_{i} c_{i}^{+} c_{i}
$$

Here $c_{i}^{+}$creates an electron at site $i$ and $w_{i}$ is the occupation number of the ions taking the value 1 or 0 at each site according to whether the site $i$ is occupied or unoccupied by an ion.

The kinetic energy (the first term of (1)) is due to quantum mechanical hopping of electrons between sites $i$ and $j$, and these intersite hopping transitions are described by the general matrix element $t_{i j}$. For the conventional Falicov-Kimball model it is usually assumed that $t_{i j}=-t$ if $i$ and $j$ are the nearest neighbors and $t_{i j}=0$ otherwise. The second term represents an on-site interaction between electrons and ions that can be repulsive $(U>0)$ or attractive $(U<0)$. In this model both the total electron number $N_{\mathrm{e}}=\sum_{i} c_{i}^{+} c_{i}$ and the total ion number $N_{i}=\sum_{i} w_{i}$ are conserved quantities.

The Falicov-Kimball model was originally introduced [1] to study mixed-valence states in rare-earth compounds. The moving particles play the role of band $s$ electrons and ions - the role of $f$ electrons. The model can be also discussed as an approximation to the full Hubbard model [2], in which only up-spin electrons are allowed to hop and down-spin electrons are infinitely massive. Moreover, it was considered by Kennedy and Lieb [3] as a model for crystallization.

In spite of its simplicity, so far only the following exact results concerning a ground state of the Hamiltonian (1) have been obtained: (1) In the symmetric case, when the total number $N_{\mathrm{e}}$ of electrons and the total number $N_{\mathrm{i}}$ of ions are equal to $\mathrm{L} / 2$ ( $\mathrm{L}$ is the number of the lattice sites) the ions form checker-board pattern [3, 4]. (2) Outside the symmetry point, the checker-board configuration, 
the completely empty and fully occupied configurations may be ground states. (3) Recently, Brandt and Mielsch [5] obtained an exact solution in $d=\infty$. (5) Freericks and Falicov [6] studied the model in one dimension (at present the exact solution of the model for $d=1$ dimension does not exist). They presented the coherent and incoherent phase diagrams calculated numerically for the segregated phase and all periodic phases with periods less than 9 and $N_{\mathrm{i}}=L / 2, L / 3$, where $L$ is the number of lattice sites. On the base of these results they formulated the conjecture, the so-called segregation principle, which states the following. In the limit $|U / t| \rightarrow \infty$ the segregated phase, which is incoherent mixture of the empty and full lattices with weights $L-N_{\mathrm{i}}$ and $N_{\mathrm{i}}$, is the ground state for all values of the electron concentration $n_{\mathrm{e}}$ except the specific values $n_{\mathrm{e}}=1-n_{\mathrm{i}}$ for $U / t \rightarrow \infty$ and $n_{\mathrm{e}}=n_{\mathrm{i}}$ for $U / t \rightarrow \infty\left(n_{\mathrm{e}}=N_{\mathrm{e}} / L, n_{\mathrm{i}}=N_{\mathrm{i}} / L\right)$. (6) Brandt [7] was the first who analytically proved that the segregation principle is true. He calculated the upper (lower) bound $U^{+}\left(U^{-}\right)$, above (below) which the segregated phase is (is not) the ground state of the one-dimensional Falicov-Kimball model. His results show that even for reasonably large deviations from the singular point $n_{\mathrm{e}} /\left(1-n_{\mathrm{i}}\right)=1$ the values $U^{+}$(this quantity is given more precisely) and $U^{-}$are extremely large.

To analyse some energetic and structural ground state properties of the Falicov-Kimball model in the strong coupling limit we use the second-order degenerate perturbation theory [8]. Let the interaction energy of the Hamiltonian (1) be the unperturbed Hamiltonian and let the kinetic energy be the perturbation. Because the matrix $W$ is idempotent, the matrix $U W$ has only two eigenvalues $E_{1}=0$ and $E_{2}=U$. They are $\left(L-N_{\mathrm{i}}\right)$-fold and $N_{\mathrm{i}}$-fold degenerate and these degeneracies in consequence of a perturbation will have been completely or partly removed. The corresponding second order corrections may be obtained using the standard perturbation theory of the degenerate levels.

For the Falicov-Kimball model this scheme leads to the following secular equation:

$$
\operatorname{det}\left(T^{\prime}-E I\right)=0,
$$

where the matrix elements $T_{n n^{\prime}}^{\prime}$ of the $N_{\mathrm{i}}$ or $L-N_{\mathrm{i}}$ square matrix $T^{\prime}$ are given by

$$
T_{n n^{\prime}}^{\prime}=t_{n n^{\prime}} \pm \frac{1}{U} \sum_{m} t_{n m} t_{m n^{\prime}}
$$

If we calculate the second order corrections to the energy level $E_{1}=0$ $\left(E_{2}=U\right)$, then $n$ and $n^{\prime}$ in (3) denote unoccupied (occupied) sites, whereas $m$ denotes occupied (unoccupied) sites and the correct sign in front of the sum is - $(+)$. (For remainder of the paper we can restrict ourselves only to the case $E_{1}=0$ and $U>0$, since other cases can be deduced from the particle-hole symmetries [6].)

Using the expression (3) we can immediately investigate the ground state phase diagram of the Falicov-Kimball model with the nearest neighbor hopping for various periodic and aperiodic configurations of the ions. For these configurations the secular equation (2) is enormously simplified and can be immediately solved. Putting electrons on the low-lying energy levels of the new spectra one can at once find energies corresponding to these configurations. The phase diagram is then determined by comparing the energies of all phases and plotting the lowest-energy 
state as a function of the electron concentration $n_{\mathrm{e}}$ and the interaction strength $U$. Although this subject is contents of our other paper [9], let us briefly discuss the phase diagram of the model for some special configurations of the ions.

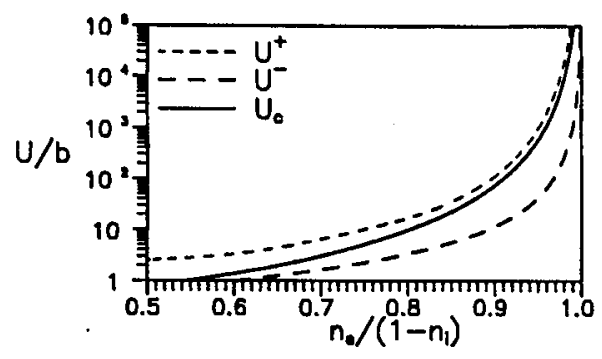

Fig. 1. The upper bound $U^{+}$and lower bound $U^{-}$from Ref. [7] and our result for lower bound $U_{c}$ (divided by the bandwidth) as a function of the electron- and ion-density ratio $n_{e} /\left(1-n_{\mathrm{i}}\right)$.

In Fig. 1 the phase diagram of the model calculated for transition $w_{S} \rightarrow w_{D}$, where $w_{S}=\{00 \ldots 011 \ldots 1\}$ is the segregated configuration and $w_{D}=\{00 \ldots 011 \ldots 1011 \ldots 1\}$, is presented. We see that there exists some critical value of the interaction strength $U_{c}$ below which the segregated phase is unstable and the large connected cluster of the unoccupied sites divides into two connected clusters of unoccupied sites. (It can be shown [10] that from among all possible divisions of the large connected cluster of unoccupied sites in the segregated configuration into two connected clusters of unoccupied sites separated, at least two ions have just the configuration $w_{D}$ with the lowest energy.) A comparison of our results for the lower bound $U^{-} \equiv U_{c}$, below which the segregated phase cannot be the ground state of the model with results of Brandt [7] shows that our estimate is much better than the estimate of Brandt, who investigated stability of the segregated phase with respect to "evaporation". But his estimate of the upper bound $U^{+}$, above which only the segregated phase is the ground state of the model is quite good for $n_{e} /\left(1-n_{\mathrm{i}}\right)>0.5$ and it yields, together with our results for $U_{c}$, very sharp bounds for the segregated statè. Of course, one can ask what happens with $w_{D}$ below $U_{\mathrm{c}}$. The answer is the following. The configuration $w_{D}$ is the ground state of the model only in the very narrow band bounded by $U_{\mathrm{c}}$ and $U_{\mathrm{c}}^{\prime}$. Below $U_{c}^{\prime}$ the configuration $w_{D}$ becomes unstable, because the largest connected cluster of unoccupied sites divides like in the preceding case, etc. Now, if we take into account the configurations of this kind (they can be represent by incoherent mixtures of the segregated configuration of the length $N$ and alternating configuration $\{1010 \ldots\}$ of the length $L-N)$, all periodic configurations with period less than 9 [6], and all periodic configurations $c_{\mathrm{i}}=\{11 \ldots 100 \ldots 0 \ldots\}$, which may be composed of smaller segregated clusters of occupied and unoccupied sites of the length $i$, we find out that for $N_{\mathrm{e}}<L-N_{\mathrm{i}}$ only these incoherent mixtures can be the ground states of the one-dimensional Falicov-Kimball model.

Finally, let us briefly discuss another interesting case, when the hopping 


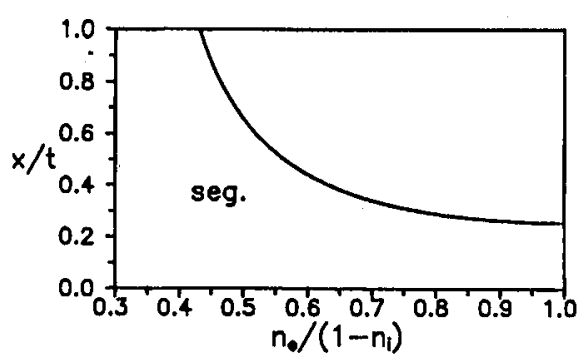

Fig. 2. The lower bound, above which the segregated phase cannot be the ground state of the one-dimensional Falicov-Kimball model with the next nearest neighbor hopping.

to the next nearest neighbors is allowed. Using the perturbation theory outlined above we found out that if the nearest neighbor hopping $(-t)$ and the next nearest neighbor hopping $(x)$ are considered simultaneously, then even for infinite $U$ the segregated principle is broken, when the ratio of $x / t$ is greater than some critical value (see Fig. 2). The fact that above this boundary the segregated phase is unstable (with respect to $w_{D}$ ) even for infinite $U$, while for $x=0$ it is the ground state of the model for all $n_{\mathrm{e}}<1-n_{\mathrm{i}}$, shows that the kinetic energy still plays dominant role.

\section{References}

[1] L.M. Falicov, J.C. Kimball, Phys. Rev. Lett. 22, 997 (1969).

[2] J. Hubbard, Proc. R. Soc. Lond. A 276, 238 (1963).

[3] T. Kennedy, E.H. Lieb, Physica A 138, 320 (1986).

[4] U. Brandt, R. Schmidt, Z. Phys. B 67, 49 (1987).

[5] U. Brandt, C. Mielsch, Z. Phys. B 75, 365 (1989).

[6] J.K. Freericks, L.M. Falicov, Phys. Rev. B 41, 2163 (1990).

[7] U. Brandt, J. Low Temp. Phys. 84, 477 (1991).

[8] L.D. Landau, E.M. Lifshitz, Quantum Mechanics, Pergamon Press, London 1977.

[9] P. Farkašovský, I. Bat'ko, J. of Physics C, Condens. Matler, 1993, submitted.

[10] P. Farkašovský, I. Bat'ko, Czech. J. Phys., 1993, accepted. 